\title{
Hypertension as a predictive biomarker in
} \section{patients with advanced non-small-cell lung cancer treated with apatinib}

This article was published in the following Dove Medical Press journal:

OncoTargets and Therapy

\author{
Shen-Cun Fang ${ }^{1,2}$ \\ Wen Huang ${ }^{2}$ \\ Ying-Ming Zhang' \\ Hai-Tao Zhang' \\ Wei-Ping $\mathrm{Xie}^{2}$ \\ 'Department of Respiratory \\ Medicine, Nanjing Chest Hospital, \\ Nanjing, Jiangsu, China; ${ }^{2}$ Department \\ of Pulmonary and Critical Care \\ Medicine, The First Affiliated Hospital \\ of Nanjing Medical University, Nanjing, \\ Jiangsu, China
}

Correspondence: Wei-Ping Xie Department of Pulmonary and Critical Care Medicine, The First Affiliated Hospital of Nanjing Medical University, 300, Guangzhou Road, Nanjing, Jiangsu, China

Tel +86 I38 5I50 68I8

Email wpxie@njmu.edu.cn
Background: Hypertension (HTN) is a common adverse event of the vascular endothelial growth factor pathway inhibitor apatinib. This study was conducted to evaluate the association of apatinib-induced HTN with clinical outcomes in patients with advanced non-small-cell lung cancer (NSCLC).

Methods: We retrospectively analyzed 110 consecutive patients with advanced NSCLC who were treated with apatinib from August 2014 to January 2018. All patients were classified as normotensive or hypertensive based on blood pressure measurements after initiating therapy. Therapeutic response, progression-free survival (PFS), and overall survival (OS) were evaluated. Univariate and multivariate analyses were performed using the Cox proportional hazards method. Results: A total of 46 patients (42\%) were diagnosed with HTN. The median PFS for the hypertensive and normotensive groups were 5.6 months and 4.2 months, respectively $(P=0.0027)$. The median OS times for the hypertensive and normotensive groups were 9.9 months and 7.8 months, respectively $(P=0.005)$. Thirty percent of patients who experienced HTN showed partial response to apatinib as compared with $6.3 \%$ of non-hypertensive patients $(P=0.002)$. HTN was independently associated with improved PFS and OS on both univariate and multivariate analyses.

Conclusion: Apatinib-induced HTN may be an inexpensive, valid, and easily measurable biomarker for apatinib antitumor efficacy in patients with advanced NSCLC.

Keywords: biomarker, hypertension, apatinib, non-small-cell lung cancer, clinical outcomes

\section{Background}

Angiogenesis plays a crucial role in the carcinogenesis, invasiveness, and metastasis of solid malignancies, while vascular endothelial growth factor (VEGF) and VEGF receptor 2 (VEGFR-2) are predominantly responsible for angiogenic signaling. ${ }^{1,2}$ Inhibition of angiogenesis is an important antitumor therapeutic strategy. Antiangiogenic agents targeting the tyrosine kinases of the VEGF receptors have demonstrated antitumor activity across a great number of malignancies. For example, vandetanib, sunitinib, and sorafenib have been approved for the treatment of thyroid cancer, ${ }^{3}$ renal cell carcinoma, ${ }^{4}$ and hepatocellular carcinoma, ${ }^{5}$ respectively. Although a large proportion of patients do not seem to benefit from antiangiogenic therapies and are therefore exposed to unnecessary toxicity, there are currently no biomarkers available to identify which patients are most likely to benefit from such a treatment approach. Thus, it is of great urgency to establish a valid and easily measurable predictive biomarker of efficacy. 
Hypertension (HTN) has been reported as a common adverse event associated with VEGF-targeted agent, although the mechanism is unclear. Antagonism of VEGF leads to reduced release of nitric oxide from endothelial cells, therefore causing vasoconstriction and increased peripheral vascular resistance, which ultimately lead to HTN. ${ }^{6,7}$ Moreover, evidence suggests that HTN may result from functional or structural vascular rarefaction induced by inhibition of VEGF pathway. ${ }^{8}$ Given that the onset of treatment-induced HTN denotes successful blockage of the VEGF signaling pathway, HTN has been proposed as a surrogate biomarker of the clinical effect of antiangiogenic agents. ${ }^{9-11}$

Apatinib, an oral small molecule tyrosine kinase inhibitor targeting VEGF receptor-2 (VEGFR-2), was approved by the China Food and Drug Administration for the treatment of advanced gastric cancer in 2014. Although apatinib was associated with a $35 \%-64.4 \%$ incidence of any-grade HTN which was reported as an adverse event in clinical trials, ${ }^{12-14}$ in a study of patients with advanced gastric cancer treated with apatinib, treatment-induced HTN significantly predicted improved clinical outcomes. ${ }^{15}$ Apatinib has also demonstrated encouraging efficiency in advanced non-small-cell lung cancer (NSCLC). Preliminary results of a Phase II clinical trial demonstrated that apatinib showed substantial clinical activity in patients with advanced non-squamous NSCLC patients. ${ }^{16}$ In combination with epidermal growth factor receptor tyrosine kinase inhibitors (EGFR-TKIs), apatinib improved outcomes for patients with advanced NSCLC after EGFR-TKI treatment failure. ${ }^{17-19}$ Apatinib has demonstrated efficacy for the treatment of advanced NSCLC when utilized as salvage treatment. ${ }^{20,21}$ We have also reported that three cases of advanced lung adenocarcinoma without identifiable driver oncogenes who received apatinib as a third- or furtherline treatment achieved partial response (PR). ${ }^{22}$

Based on these observations, a retrospective analysis was performed to investigate whether the development of HTN in apatinib-treated patients with advanced NSCLC was associated with clinical outcomes. The purpose of this study was to determine whether apatinib-induced HTN could predict longer OS in advanced NSCLC patients and serve as a biomarker of efficacy.

\section{Methods}

\section{Patients}

Consecutive patients with unresectable stage IV NSCLC treated with apatinib were enrolled between August 2014 and January 2018 in Nanjing Chest Hospital and the First Affiliated Hospital of Nanjing Medical University. Key inclusion criteria for patients were histologically confirmed advanced NSCLC, at least one measurable lesion according to Response Evaluation Criteria in Solid Tumors (RECIST), Eastern Cooperative Oncology Group performance status (ECOG PS) $0-3$, and sufficiently recorded source data. Exclusion criteria included patients with uncontrolled HTN (>140/90 mmHg) and those with thrombotic or hemorrhagic disorders. One hundred ten patients were finally included in this analysis. All patients underwent treatment with apatinib monotherapy. The starting dose of apatinib monotherapy was $500 \mathrm{mg}$ per day and the dose should be reduced to $250 \mathrm{mg}$ if there is an untolerated toxicity. This study was approved by the Medical Ethics Committee of Nanjing Chest Hospital and all aspects of the study comply with the Declaration of Helsinki guidelines. The Ethics Committee of Nanjing Chest Hospital specifically approved that no informed consent was required because it was a retrospective study. Data of all patients were confidential at the strictest level. All participants in the study were completely voluntary and not forced to participate.

\section{Study assessments}

The therapy response was evaluated according to RECIST criteria by performing computed tomography (CT) and/ or magnetic resonance imaging (MRI) every 8 weeks until disease progression. Complete response (CR) means disappearance of all target lesions. PR means the longest diameter of target lesion was reduced by at least $30 \%$. Stable disease (SD) means the longest diameter of the target lesion increased to less than progressive disease (PD), or reduced to less than PR. PD means that the longest diameter of the target lesion increased by at least $20 \%$, or the appearance of new lesion. Progression-free survival (PFS) was defined as the time from the first administration of apatinib to the date of disease progression. Overall survival (OS) was defined as the time from the first administration of apatinib to death or loss of follow-up. HTN was defined as maximum systolic pressure $\geq 140 \mathrm{mmHg}$ and/or diastolic pressure $\geq 90 \mathrm{mmHg}$ at any time after day 1 of treatment. HTN was defined and graded according to the Common Toxicity Criteria for Adverse Events version 4.0. ${ }^{23}$ In all patients with elevated baseline HTN, blood pressure (BP) should be well controlled before receiving apatinib therapy.

\section{Statistical analysis}

Chi-squared test or Fisher's exact test was used to assess the association between categorical variables. PFS and OS were assessed using the Kaplan-Meier method and compared between patients with and without HTN using the log-rank test. The influence of covariates on PFS and OS was assessed using a Cox proportional hazards model. The variables in 
univariate analysis with $P<0.2$ were entered in the multivariable model. The purpose of the landmark analysis was to avoid the possible guarantee-time bias caused by incorrectly classifed patients in the normotensive group if they had died or discontinued taking apatinib before experiencing the HTN. Moreover, guarantee-time bias also included the possibility that patients developed inauthentic HTN long after apatinib treatment, which cannot be interpreted as apatinib-induced HTN. Landmark analyses were performed using KaplanMeier methods at the end of 2 months (the 95th percentile time for the occurrence of HTN). Therefore, patients who develop HTN after 2 months should be excluded from landmark analysis in the hypertensive group, and patients who stopped taking apatinib or died before 2 months were also not included. Two-sided tests were used, and a $P$-value $<0.05$ was considered statistically significant. Statistical analyses were performed using SPSS version 19.0.

\section{Results}

\section{Patient characteristics}

The demographic characteristics of 110 patients with advanced NSCLC included in this analysis are summarized in Table 1. The median age of patients was 65 years (range 44-81 years). A total of 46 patients (42\%) were diagnosed with HTN (grade $1[\mathrm{n}=14]$, grade $2[\mathrm{n}=22]$, and grade 3 $[n=10])$, whereas the rest of the patients (58\%) did not develop HTN during apatinib treatment. No patient developed HTN grade 4 or grade 5 . There were no significant differences in age, gender, smoking history, ECOG PS, pathological type, location, and line of apatinib treatment between the normotensive and hypertensive patients.

\section{Effect of HTN on clinical outcomes}

The onset of HTN was significantly correlated with improved outcomes (Table 2). The median PFS for all patients was 4.6 months. The median PFS for patients with apatinibinduced HTN was 5.6 months, and for normotensive patients, it was 4.2 months $(P=0.0027)$ (Figure 1A). The median OS for all patients was 8.2 months. Again, patients with apatinib-induced HTN had a significantly prolonged OS, with a median OS of 9.9 months reported in the hypertensive patients and 7.8 months noted in the normotensive patients $(P=0.005)$ (Figure 1B). Furthermore, HTN was associated with significantly better responses to therapy. Thirty percent of patients who experienced HTN showed PR to apatinib as compared with $6.3 \%$ of non-hypertensive patients $(P=0.002)$. None achieved a $\mathrm{CR}$ in both the normotensive and hypertensive groups (Table 2).

Table I Patient characteristics

\begin{tabular}{|c|c|c|c|}
\hline Characteristics & $\begin{array}{l}\text { Normotensive } \\
(\mathrm{N}=64), \mathrm{N}(\%)\end{array}$ & $\begin{array}{l}\text { Hypertensive } \\
(\mathrm{N}=46), \mathrm{N}(\%)\end{array}$ & $P$-value \\
\hline Median age (range), years & $59(52-8 I)$ & $67(44-8 I)$ & 0.089 \\
\hline Gender & & & 0.475 \\
\hline Female & $15(24.1)$ & $15(33.3)$ & \\
\hline Male & $49(75.9)$ & $31(66.7)$ & \\
\hline Smoking history & & & 0.963 \\
\hline Smoker & $51(5 \mathrm{I} .7)$ & $24(52.4)$ & \\
\hline Non-smoker & $13(48.3)$ & $22(47.6)$ & \\
\hline ECOG PS & & & 0.152 \\
\hline $0-1$ & $37(58.6)$ & $18(38.1)$ & \\
\hline$\geq 2$ & $27(4 \mid .4)$ & $28(6 \mid .9)$ & \\
\hline Pathological type & & & 0.307 \\
\hline Adenocarcinoma & $40(62.1)$ & $28(6 \mid .9)$ & \\
\hline Squamous carcinoma & $7(10.3)$ & II (23.8) & \\
\hline Other & $17(27.6)$ & $7(14.3)$ & \\
\hline Location & & & 0.603 \\
\hline Central & $20(3 I)$ & $18(38.1)$ & \\
\hline Peripheral & $44(69)$ & $28(6 \mid .9)$ & \\
\hline Line of apatinib & & & 0.352 \\
\hline Second line & $26(4 \mid .4)$ & $13(28.6)$ & \\
\hline Further line & $38(58.6)$ & 33 (7I.4) & \\
\hline
\end{tabular}

Abbreviation: ECOG PS, Eastern Cooperative Oncology Group performance status. 
Table 2 Treatment efficacy in patients with and without HTN

\begin{tabular}{|l|l|l|l|l|}
\hline Clinical outcomes & $\begin{array}{l}\text { All } \\
(\mathbf{n = I} \mathbf{0})\end{array}$ & $\begin{array}{l}\text { Normotensive } \\
(\mathbf{n = 6 4 )}\end{array}$ & $\begin{array}{l}\text { Hypertensive } \\
(\mathbf{n = 4 6 )}\end{array}$ & P-value \\
\hline Response rate & & & & 0.002 \\
\hline PR & 18 & 4 & 14 & \\
\hline SD & 55 & 31 & 24 & \\
\hline PD & 37 & 29 & 8 & \\
\hline PFS (months) & 4.6 & 4.2 & 5.6 & 0.0027 \\
\hline OS (months) & 8.2 & 7.8 & 9.9 & 0.005 \\
\hline
\end{tabular}

Abbreviations: HTN, hypertension; PR, partial response; SD, stable disease; PD, progressive disease; PFS, progression-free survival; OS, overall survival.

\section{Univariate and multivariate analyses}

In univariate analysis, PS $(P=0.029)$, age $(P=0.037)$, and HTN $(P=0.0027)$ were obviously associated with PFS (Table 3). In multivariate analysis, patients with good PS $(P=0.017$, HR: 2.26 , CI: $1.16-4.39)$ and HTN $(P=0.011$, HR: 0.413, CI: $0.21-0.82)$ had significantly longer PFS. Moreover, the apatinib-induced HTN was also found to be independently predictive of improved OS on both univariate and multivariate analyses (Table 4).

\section{Landmark analysis}

To address possible guarantee-time bias from misclassification of patients, landmark analyses were performed at the end of 2 months. After adjusting for potential bias, the median OS was 10.2 months (CI: 7.9-11.4) for the patients with HTN vs 8.6 months (CI: 6.17-9.43) for the normotensive patients $(P=0.008)$ (Figure 2). The adjusted HR for OS of patients with HTN was 0.28 (CI: 0.15-0.37).

\section{Discussion}

This retrospective analysis found that HTN was associated with significantly improved clinical outcomes in patients with

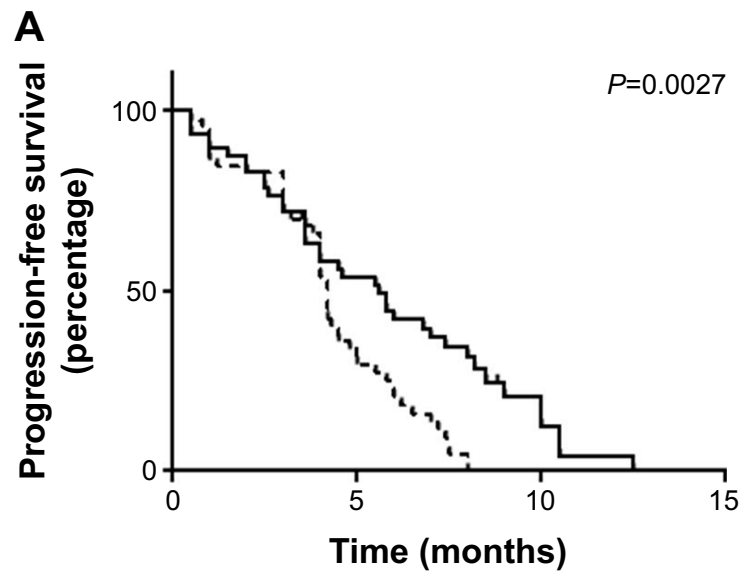

advanced NSCLC treated with apatinib. These results support the hypothesis that HTN may be a predictive biomarker of efficacy for apatinib treatment in this patient population. Response rate was more than five times greater in patients with HTN than in those without HTN. Moreover, Median PFS and OS were significantly longer among patients with HTN than those without. Similarly, results of landmark analyses demonstrated that HTN onset by the end of the second treatment cycle was also associated with improved outcomes. Finally, HTN was identified as an independent prognostic factor for improved survival in both univariate and multivariate analyses.

Indeed, a number of previous studies have evidenced HTN as a surrogate biomarker for efficacy of antiangiogenic therapy. In line with our results, in a cohort of 80 patients treated with apatinib for advanced breast cancer, Fan et al demonstrated that HTN was correlated with significant benefits in terms of response rate, PFS, and OS. ${ }^{24}$ In addition, retrospective analyses evaluating the relationship between HTN and outcomes in advanced NSCLC patients treated with bevacizumab or axitinib showed a similar result to that observed here for apatinib. ${ }^{25,26}$ These observations,

B

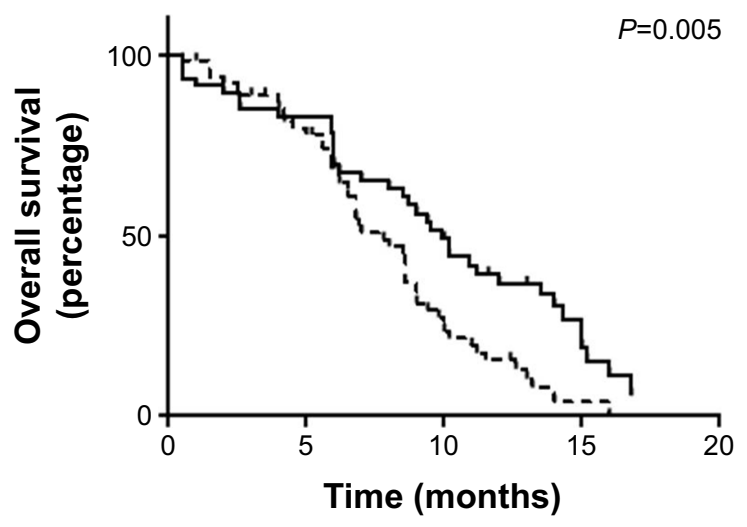

\section{- ᄂ. Normotensive $ـ$ Hypertensive}

Figure I Overall survival and progression-free survival by hypertension during apatinib treatment.

Notes: (A) PFS was compared between the two groups. (B) OS was compared between the two groups. 
Table 3 Progression-free survival in univariate and multivariate analyses

\begin{tabular}{|c|c|c|c|}
\hline \multirow[t]{2}{*}{ Variables } & \multirow[t]{2}{*}{ Median $(95 \% \mathrm{Cl})$} & \multicolumn{2}{|l|}{$P$-value } \\
\hline & & Univariate & Multivariate \\
\hline Age (years) & & 0.037 & 0.089 (HR: $0.46,95 \% \mathrm{Cl}: 0.2 \mathrm{I}-\mathrm{I} . \mathrm{I} 2)$ \\
\hline$\geq 65$ & $5.3(3.59-7.01)$ & & \\
\hline$<65$ & $3.8(2.4 \mid-5.19)$ & & \\
\hline Gender & & 0.422 & \\
\hline Female & $4.2(3.25-5.15)$ & & \\
\hline Male & $4.6(3.52-5.68)$ & & \\
\hline Smoking history & & 0.178 & \\
\hline Smoker & $5(3.55-6.45)$ & & \\
\hline Non-smoker & $4(I .49-6.5 I)$ & & \\
\hline ECOG performance status & & 0.029 & 0.017 (HR: 2.26, 95\% Cl: I.I6-4.39) \\
\hline $0-1$ & $5.5(4.26-6.74)$ & & \\
\hline$\geq 2$ & $3.6(2.47-4.73)$ & & \\
\hline Pathological type & & 0.53 & \\
\hline Adenocarcinoma & $4.2(3.56-4.84)$ & & \\
\hline Non-adenocarcinoma & $5(3.78-6.22)$ & & \\
\hline Line of apatinib & & 0.212 & \\
\hline Second line & $5.3(4.34-6.26)$ & & \\
\hline Further line & $3.6(3.1-4.1)$ & & \\
\hline Location & & 0.515 & \\
\hline Central & $4(2.23-5.77)$ & & \\
\hline Peripheral & $4.6(3.76-5.44)$ & & \\
\hline Apatinib-induced hypertension & & 0.0027 & $0.01 \mathrm{I}$ (HR: $0.4 \mathrm{I}, 95 \% \mathrm{Cl}: 0.2 \mathrm{I}-0.82)$ \\
\hline Yes & $5.6(3.75-6.85)$ & & \\
\hline No & $4.2(3.28-5.12)$ & & \\
\hline
\end{tabular}

Abbreviation: ECOG, Eastern Cooperative Oncology Group.

if validated by large-scale prospective studies, seem to imply that more active attempts should be used to control BP rather than reduction in drug dosing or interruption in treatment when severe HTN occurs. Aggressive BP monitoring and treatment for HTN with antihypertensive medication in patients receiving VEGF inhibitors were recommended by an expert panel of the National Cancer Institute. ${ }^{27}$ Antihypertensive drugs should be regarded as first-line treatment of apatinib-induced HTN, considering the results that use of antihypertensive agents did not reduce antitumor activity of VEGF inhibitors. ${ }^{28}$ Conversely, the lack of HTN may suggest an early change in the treatment strategy. Rini et al proposed that upward dose titration of axitinib until BP elevation in an individual patient might lead to superior outcomes. ${ }^{29}$ Initial observations in an apatinib Phase I trial demonstrated the dose-dependent relationship between apatinib and HTN induction. ${ }^{30}$ Thus, dosage titration of apatinib for individual patients may be crucial to increasing responses. However, these methods must be confirmed in clinical studies before they can be applied.
A large number of studies indicate that genetic variations in HTN-associated genes are associated with the risk for developing antiangiogenic drug-induced HTN. In this setting, dosage titration of apatinib may not achieve the expected effect when patients harboring genetic variations in HTN-associated genes are naturally more resistant to drug-induced HTN despite sufficient drug concentration. Melissa et al evaluated patients with solid tumors treated with bevacizumab in six clinical trials and found that both single-nucleotide polymorphisms (SNPs) and haplotypes in WNK1 and KLKB1 were associated with the development of bevacizumab-induced HTN, whereas three haplotypes in GRK4 were related to protection from HTN. ${ }^{31}$ Morita et al reported an association between the VEGF-2578 C/C genotype and less HTN in 60 Japanese patients treated with bevacizumab-based chemotherapy. ${ }^{32}$ SNPs in VEGF (VEGF634 CC and VEGF-1498 TT) have also been identified to be associated with protection from grade $3 / 4 \mathrm{HTN} .{ }^{33}$ If there is a definite link between HTN and effectiveness of the apatinib in the patient cohort in the present study, then detection of 
Table 4 Overall survival in univariate and multivariate analyses

\begin{tabular}{|c|c|c|c|}
\hline \multirow[t]{2}{*}{ Variables } & \multirow[t]{2}{*}{ Median $(95 \% \mathrm{Cl})$} & \multicolumn{2}{|l|}{$P$-value } \\
\hline & & Univariate & Multivariate \\
\hline Age (years) & & 0.045 & 0.092 (HR: $0.67,95 \%$ Cl: $0.12-1.22)$ \\
\hline$\geq 65$ & $9.2(8.25-10.15)$ & & \\
\hline$<65$ & $6.6(5.66-7.54)$ & & \\
\hline Gender & & 0.704 & \\
\hline Female & $8.2(7.62-8.78)$ & & \\
\hline Male & $8.2(6.13-10.27)$ & & \\
\hline Smoking history & & 0.999 & \\
\hline Smoker & $8.2(6.05-10.35)$ & & \\
\hline Non-smoker & $8.8(7.8-9.8)$ & & \\
\hline ECOG performance status & & 0.119 & \\
\hline $0-1$ & $9.4(7.9-10.87)$ & & \\
\hline$\geq 2$ & $8(6.0 I-9.99)$ & & \\
\hline Pathological type & & 0.364 & \\
\hline Adenocarcinoma & $8(6.37-9.63)$ & & \\
\hline Non-adenocarcinoma & $9.4(8.22-10.58)$ & & \\
\hline Line of apatinib & & 0.123 & \\
\hline Second line & $9.4(6.62-12.18)$ & & \\
\hline Further line & $8.2(5.93-10.47)$ & & \\
\hline Location & & 0.971 & \\
\hline Central & $8.2(6.28-10.12)$ & & \\
\hline Peripheral & $8.5(7.48-9.52)$ & & \\
\hline Apatinib-induced hypertension & & 0.005 & 0.022 (HR: $0.45,95 \%$ Cl: $0.23-0.89)$ \\
\hline Yes & $9.9(7.9-10.8)$ & & \\
\hline No & $7.8(4.98-8.62)$ & & \\
\hline
\end{tabular}

Abbreviation: ECOG, Eastern Cooperative Oncology Group.

HTN protective genes will be contributing to screen out patients with natural resistance to apatinib. But to date, none have been validated.

There are several limitations in the current study. First, the small sample size and retrospective design of the present study may bias the result. Future large-scale prospective studies

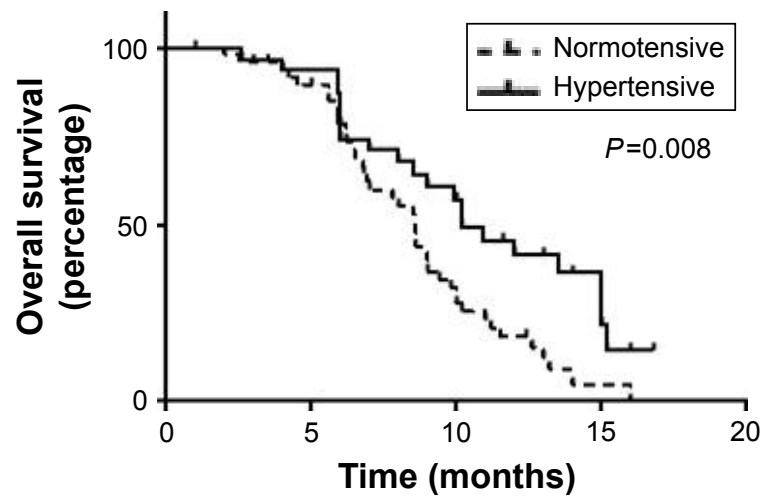

Figure 2 Landmark analysis of overall survival in patients with and without hypertension with landmark at 2 months. should be conducted to confirm the findings of this study. Second, our definition of HTN only refers to an increase in the threshold level of systolic and/or diastolic pressure, which may not be accurate to characterizing apatinib-induced HTN. VEGF inhibitor-induced HTN was defined by other studies as both BP increase from baseline and start or extension of antihypertensive medication. ${ }^{10,34}$ However, no studies have confirmed that one definition is superior to the other as a biomarker of clinical outcomes. Finally, the management of side effects like HTN was not uniform in each patient, which may affect the prognosis of patients.

\section{Conclusion}

Despite substantial efforts, till date no validated predictive biomarkers for antiangiogenic agents have been identified. Serial BP monitoring is convenient and may be reflective of intended target inhibition once therapy is started. Results of this retrospective analysis suggest that treatment-induced HTN may be an inexpensive, valid, and easily measurable 
biomarker for apatinib antitumor efficacy in patients with advanced NSCLC. Therefore, larger randomized studies should be performed to evaluate the role of apatinib-induced HTN as a potential prognostic biomarker in advanced NSCLC patients treated with apatinib.

\section{Acknowledgment}

This study was supported by the Priority Academic Program Development of Jiangsu Higher Education Institutions (PAPD) and Jiangsu Provincial Youth Medical Talent Program (QNRC2016123).

\section{Author contributions}

All authors contributed toward data analysis, drafting, and critically revising the paper; gave final approval of the version to be published; and agree to be accountable for all aspects of the work.

\section{Disclosure}

The authors report no conflicts of interest in this work.

\section{References}

1. Hicklin DJ, Ellis LM. Role of the vascular endothelial growth factor pathway in tumor growth and angiogenesis. J Clin Oncol. 2005;23(5): 1011-1027.

2. Atzori MG, Tentori L, Ruffini F, et al. The anti-vascular endothelial growth factor receptor-1 monoclonal antibody D16F7 inhibits invasiveness of human glioblastoma and glioblastoma stem cells. $J$ Exp Clin Cancer Res. 2017;36(1):106.

3. Wells SA, Gosnell JE, Gagel RF, et al. Vandetanib for the treatment of patients with locally advanced or metastatic hereditary medullary thyroid cancer. J Clin Oncol. 2010;28(5):767-772.

4. Motzer RJ, Hutson TE, Tomczak P, et al. Sunitinib versus interferon alfa in metastatic renal-cell carcinoma. $N$ Engl J Med. 2007;356(2): $115-124$.

5. Llovet JM, Ricci S, Mazzaferro V, et al. Sorafenib in advanced hepatocellular carcinoma. N Engl J Med. 2008;359(4):378-390.

6. Sane DC, Anton L, Brosnihan KB. Angiogenic growth factors and hypertension. Angiogenesis. 2004;7(3):193-201.

7. Bhargava P. VEGF kinase inhibitors: how do they cause hypertension? Am J Physiol Regul Integr Comp Physiol. 2009;297(1):R1-R5.

8. Mourad JJ, des Guetz G, Debbabi H, Levy BI. Blood pressure rise following angiogenesis inhibition by bevacizumab. A crucial role for microcirculation. Ann Oncol. 2008;19(5):927-934.

9. Goodwin R, Ding K, Seymour L, et al. Treatment-emergent hypertension and outcomes in patients with advanced non-small-cell lung cancer receiving chemotherapy with or without the vascular endothelial growth factor receptor inhibitor cediranib: NCIC Clinical Trials Group study BR24. Ann Oncol. 2010;21(11):2220-2226.

10. Zhong J, Ali AN, Voloschin AD, et al. Bevacizumab-induced hypertension is a predictive marker for improved outcomes in patients with recurrent glioblastoma treated with bevacizumab. Cancer. 2015;121(9): 1456-1462.

11. Jain L, Sissung TM, Danesi R, et al. Hypertension and hand-foot skin reactions related to VEGFR2 genotype and improved clinical outcome following bevacizumab and sorafenib. J Exp Clin Cancer Res. 2010; 29(1):95.
12. Li J, Qin S, Xu J, et al. Randomized, double-blind, placebo-controlled phase III trial of apatinib in patients with Chemotherapy-Refractory advanced or metastatic adenocarcinoma of the stomach or gastroesophageal junction. J Clin Oncol. 2016;34(13):1448-1454.

13. $\mathrm{Hu} \mathrm{X}, \mathrm{Cao} \mathrm{J}, \mathrm{Hu} \mathrm{W}$, et al. Multicenter phase II study of apatinib in nontriple-negative metastatic breast cancer. BMC Cancer. 2014;14(1):820

14. Hu X, Zhang J, Xu B, et al. Multicenter phase II study of apatinib, a novel VEGFR inhibitor in heavily pretreated patients with metastatic triple-negative breast cancer. Int J Cancer. 2014;135(8):1961-1969.

15. Liu X, Qin S, Wang Z, et al. Early presence of anti-angiogenesisrelated adverse events as a potential biomarker of antitumor efficacy in metastatic gastric cancer patients treated with apatinib: a cohort study. J Hematol Oncol. 2017;10(1):153.

16. Zhang L, Shi M, Huang C, Liu X. A phase II, multicenter, placebocontrolled trial of apatinib in patients with advanced nonsquamous non-small cell lung cancer (NSCLC) after two previous treatment regimens. J Clin Oncol. 2012;15(Suppl):7548.

17. Xu J, Liu X, Yang S, Zhang X, Shi Y. Apatinib plus icotinib in treating advanced non-small cell lung cancer after icotinib treatment failure: a retrospective study. Onco Targets Ther. 2017;10:4989-4995.

18. Li F, Zhu T, Cao B, Wang J, Liang L. Apatinib enhances antitumour activity of EGFR-TKIs in non-small cell lung cancer with EGFR-TKI resistance. Eur J Cancer. 2017;84:184-192.

19. Peng Y, Cui H, Liu Z, et al. Apatinib to combat EGFR-TKI resistance in an advanced non-small cell lung cancer patient with unknown EGFR status: a case report. Onco Targets Ther. 2017;10:2289-2295.

20. Song Z, Yu X, Lou G, Shi X, Zhang Y. Salvage treatment with apatinib for advanced non-small-cell lung cancer. Onco Targets Ther. 2017;10: 1821-1825.

21. Zeng DX, Wang CG, Lei W, Huang JA, Jiang JH. Efficiency of low dosage apatinib in post-first-line treatment of advanced lung adenocarcinoma. Oncotarget. 2017;8(39):66248-66253.

22. Fang SC, Zhang HT, Zhang YM, Xie WP. Apatinib as post second-line therapy in EGFR wild-type and ALK-negative advanced lung adenocarcinoma. Onco Targets Ther. 2017;10:447-452.

23. National Cancer Institute. Common Terminology Criteria for Adverse Events, Version 4.0. Bethesda, MD: National Cancer Institute, National Institutes of Health, Department of Health and Human Services; 2009.

24. Fan M, Zhang J, Wang Z, et al. Phosphorylated VEGFR2 and hypertension: potential biomarkers to indicate VEGF-dependency of advanced breast cancer in anti-angiogenic therapy. Breast Cancer Res Treat. 2014; 143(1):141-151.

25. Dahlberg SE, Sandler AB, Brahmer JR, Schiller JH, Johnson DH Clinical course of advanced non-small-cell lung cancer patients experiencing hypertension during treatment with bevacizumab in combination with carboplatin and paclitaxel on ECoG 4599. J Clin Oncol. 2010; 28(6):949-954.

26. Rini BI, Schiller JH, Fruehauf JP, et al. Diastolic blood pressure as a biomarker of axitinib efficacy in solid tumors. Clin Cancer Res. 2011; 17(11):3841-3849.

27. Maitland ML, Bakris GL, Black HR, et al. Initial assessment, surveillance, and management of blood pressure in patients receiving vascular endothelial growth factor signaling pathway inhibitors. J Natl Cancer Inst. 2010;102(9):596-604.

28. Rini BI, Cohen DP, Lu DR, et al. Hypertension as a biomarker of efficacy in patients with metastatic renal cell carcinoma treated with sunitinib. J Natl Cancer Inst. 2011;103(9):763-773.

29. Rini BI, Melichar B, Ueda T, et al. Axitinib with or without dose titration for first-line metastatic renal-cell carcinoma: a randomised double-blind phase 2 trial. Lancet Oncol. 2013;14(12):1233-1242.

30. Li J, Zhao X, Chen L, et al. Safety and pharmacokinetics of novel selective vascular endothelial growth factor receptor-2 inhibitor YN968D1 in patients with advanced malignancies. BMC Cancer. 2010;10(1):529.

31. Lambrechts D, Moisse M, Delmar P, et al. Genetic markers of bevacizumab-induced hypertension. Angiogenesis. 2014;17(3): 685-694. 
32. Morita S, Uehara K, Nakayama G, et al. Association between bevacizumab-related hypertension and vascular endothelial growth factor (VEGF) gene polymorphisms in Japanese patients with metastatic colorectal cancer. Cancer Chemother Pharmacol. 2013;71(2):405-411.

33. Schneider BP, Wang M, Radovich M, et al; ECOG 2100. Association of vascular endothelial growth factor and vascular endothelial growth factor receptor-2 genetic polymorphisms with outcome in a trial of paclitaxel compared with paclitaxel plus bevacizumab in advanced breast cancer: ECoG 2100. J Clin Oncol. 2008;26(28):4672-4678.
34. Gampenrieder SP, Romeder F, Muß C, et al. Hypertension as a predictive marker for bevacizumab in metastatic breast cancer: results from a retrospective matched-pair analysis. Anticancer Res. 2014;34(1): $227-233$.

\section{Publish your work in this journal}

OncoTargets and Therapy is an international, peer-reviewed, open access journal focusing on the pathological basis of all cancers, potential targets for therapy and treatment protocols employed to improve the management of cancer patients. The journal also focuses on the impact of management programs and new therapeutic agents and protocols on

\section{Dovepress}

patient perspectives such as quality of life, adherence and satisfaction The manuscript management system is completely online and includes a very quick and fair peer-review system, which is all easy to use. Visit http://www.dovepress.com/testimonials.php to read real quotes from published authors.

Submit your manuscript here: http://www.dovepress.com/oncotargets-and-therapy-journal 